\title{
SÉANCE DU 8 OCTOBRE 1920
}

\author{
PRÉSIDENGE DE M. BOIS.
}

M. le Président a le regret d'annoncer le décès de deux de nos confrères : MM. de Kersers et Burnat.

Depuis la mort de Em. Boudier, le dernier des fondateurs de la Société, M. Burnat était le doyen de nos membres et par l'áge et par la date de son admission dans notre Société, à laquelle il a donné tant de preuves de lintérêt qu'il lui portait. Une notice nécrologique sera ultérieurement publiée sur notre éminent confrère par M. John Briquet. tation.

M. le Président annonce ensuite une nouvelle présen-

MM. Gattefossé, de Puymaly, Debaire et Heim, récemment admis, ont adressé des lettres de remerciements à la Société.

M. le Secrétaire général donne un compte rendu sommaire de la session extraordinaire tenue en juillet dernier par la Société en Savoie. Il annonce qu'ún projet de session au Maroc en 1921 est à l'étude.

M. Souèges fait la communication suivante :

\section{Développement de l'embryon chez le Ghenopodium Bonus-Henricus L.; \\ PAR Y. R. SOUÉGES.}

Mes précédentes investigations sur l'embryogénie des Polygonacées ${ }^{1}$ m'ont conduit à des résultats dont l'intérêt ne pouvait réellement être déterminé qu'en recherchant dans quelle

1. Voir ce Bulletin, LXVI, p. 168, 1919.

r. Lxvir.

(SÉANCES) 16 


\section{$2 \mathrm{BHL}$ Biodiversity Heritage Library}

1920. "Séance Du 8 Octobre 1920." Bulletin de la Société botanique de France 67, 233-233. https://doi.org/10.1080/00378941.1920.10836151.

View This Item Online: https://www.biodiversitylibrary.org/item/8687

DOI: https://doi.org/10.1080/00378941.1920.10836151

Permalink: https://www.biodiversitylibrary.org/partpdf/161171

\section{Holding Institution}

Missouri Botanical Garden, Peter H. Raven Library

\section{Sponsored by}

Missouri Botanical Garden

\section{Copyright \& Reuse}

Copyright Status: Public domain. The BHL considers that this work is no longer under copyright protection.

This document was created from content at the Biodiversity Heritage Library, the world's largest open access digital library for biodiversity literature and archives. Visit BHL at https://www.biodiversitylibrary.org. 